PROCEEDINGS OF THE

AMERICAN MATHEMATICAL SOCIETY

Volume 138, Number 10, October 2010, Pages 3551-3567

S 0002-9939(10)10384-0

Article electronically published on April 16, 2010

\title{
AN OPTIMIZATION PROBLEM \\ FOR THE FIRST WEIGHTED EIGENVALUE PROBLEM \\ PLUS A POTENTIAL
}

\author{
LEANDRO M. DEL PEZZO AND JULIÁN FERNÁNDEZ BONDER \\ (Communicated by Chuu-Lian Terng)
}

\begin{abstract}
In this paper, we study the problem of minimizing the first eigenvalue of the $p$-Laplacian plus a potential with weights when the potential and the weight are allowed to vary in the class of rearrangements of a given fixed potential $V_{0}$ and weight $g_{0}$. Our results generalize those obtained in earlier papers.
\end{abstract}

\section{INTRODUCTION}

In this paper we consider the following nonlinear eigenvalue problem with weights:

$$
\begin{cases}-\Delta_{p} u+V(x)|u|^{p-2} u=\lambda g(x)|u|^{p-2} u & \text { in } \Omega, \\ u=0 & \text { on } \partial \Omega,\end{cases}
$$

where $\Omega$ is a smooth bounded open subset of $\mathbb{R}^{N}$. Here $\Delta_{p} u:=\operatorname{div}\left(|\nabla u|^{p-2} \nabla u\right)$ is the well-known $p$-Laplace operator, $V$ is a potential function and $g$ is a weight.

Our aim is to study the following optimization problems:

$$
I:=\inf \left\{\lambda(g, V): g \in \mathcal{R}\left(g_{0}\right), V \in \mathcal{R}\left(V_{0}\right)\right\}
$$

where $V_{0}$ and $g_{0}$ are fixed potential and weight functions respectively with some precise hypotheses that we state below (see (H1) and (H2)) and $\mathcal{R}\left(V_{0}\right), \mathcal{R}\left(g_{0}\right)$ are the classes of rearrangements of $V_{0}$ and $g_{0}$ respectively.

Optimization problems of this type for eigenvalues of the $p$-Laplacian have deserved a great deal of attention. We would like to mention the work of [1, where the problem was analyzed in the context of the classical Laplacian $(p=2)$ without weights $(g \equiv 1)$ and the potential was allowed to vary in the unit ball of some $L^{q}(\Omega)$.

Later on, the results in [1] were extended to the nonlinear case in 9], again without weights.

Received by the editors June 16, 2009 and, in revised form, October 30, 2009 and December 21, 2009.

2000 Mathematics Subject Classification. Primary 49K20, 35P15, 35J10.

Key words and phrases. Optimization, nonlinear eigenvalues, rearrangements.

The first author is a fellow of CONICET.

The second author was supported by Universidad de Buenos Aires under grant X078 and by ANPCyT PICT2006-290. He is a member of CONICET.

(C)2010 American Mathematical Society Reverts to public domain 28 years from publication 
A related minimization problem when the minimization parameter was allowed to vary in the class of rearrangements of a fixed function was first considered by [4. See also [8].

The eigenvalue problem (1.1) was analyzed exhaustively in [7, where the authors prove the existence of a principal eigenvalue and several properties of it. The results of 7 that are closely related to our work are discussed in Section 2.

More recently, in [5], the authors analyze problem (1.2) but when the potential function is zero. In that work the authors prove the existence of a minimizing weight $g_{*}$ in the class of rearrangements of a fixed function $g_{0}$ and, in the spirit of 2], they find a sort of Euler-Lagrange formula for $g_{*}$. However, this formula does not appear to be suitable for use in actual computations of these minimizers.

In this work we first extend the results in [5 to (1.1) and prove the existence of a minimizing weight and potential for (1.2). Also the same type of Euler-Lagrange formula is proved for both the weight and potential. But we go further and study the dependence of the eigenvalue $\lambda(g, V)$ with respect to $g$ and $V$ and prove the continuous dependence in the $L^{q}$-norm and, moreover, the differentiability with respect to regular perturbations of the weight and the potential.

In the case when the perturbations are made inside the class of rearrangements, we exhibit a simple formula for the derivative of the eigenvalue with respect to $g$ and $V$.

We believe that this formula can be used in actual computations of the optimal eigenvalue, weight and potential, since formulas of this type have been used in similar problems in the past with significant success; see [10, 11, 13, 14] and the references therein. This is what we think is the main contribution of our paper.

Organization of the paper. After finishing this introduction, the paper is organized as follows. In Section 2 we collect some preliminaries needed in the paper. First we discuss the results of [6, 7] on the eigenvalue problem (1.1) and second we recall some known results on rearrangements due to [2, 3. In Section 3 we prove the existence of a unique minimizer and give a characterization of it, similar to the one found in [5] for the problem without potential. In Section 4 we study the dependence of the eigenvalue with respect to the weight and the potential and prove, first the continuous dependence in the $L^{q}$ topology (Proposition 4.1). Finally, we show a simple formula for the derivative of the eigenvalue with respect to regular variations of the weight and the potential within the class of rearrangements (Theorem 4.12).

\section{Preliminaries}

2.1. Properties of the principal eigenvalue. Let $\Omega$ be a bounded smooth domain in $\mathbb{R}^{N}$ with $N \geq 2$ and $1<p<\infty$. Let $g_{0}$ and $V_{0}$ be measurable functions that satisfy the following assumptions:

$$
\begin{aligned}
& g_{0}, V_{0} \in L^{q}(\Omega), \text { where } \begin{cases}q>\frac{N}{p} & \text { if } 1<p \leq N, \\
q=1 & \text { if } p>N,\end{cases} \\
& \left\|V_{0}^{-}\right\|_{L^{q}(\Omega)}<S_{p q^{\prime}} \text { or } V_{0} \geq-S_{p}+\delta \text { for some } \delta>0 \text { and } g_{0}^{+} \not \equiv 0,
\end{aligned}
$$


where $f^{-}=\min \{f, 0\}, f^{+}=\max \{f, 0\}$ and $S_{r}\left(r=p, p q^{\prime}\right)$ is the best (largest) constant in the Sobolev-Poincaré inequality

i.e.,

$$
S\|u\|_{L^{r}(\Omega)}^{p} \leq \int_{\Omega}|\nabla u|^{p} \mathrm{~d} x \quad \forall u \in W_{0}^{1, p}(\Omega)
$$

$$
S_{r}:=\inf \left\{\int_{\Omega}|\nabla u|^{p} \mathrm{~d} x: u \in W_{0}^{1, p}(\Omega),\|u\|_{L^{r}(\Omega)}=1\right\} .
$$

Observe that if $g$ and $V$ are measurable functions that satisfy (H1) and (H2), then there exists a unique positive principal eigenvalue $\lambda(g, V)$ of (1.1) characterized by $(2.1)$

$$
\lambda(g, V):=\min \left\{\int_{\Omega}|\nabla u|^{p}+V(x)|u|^{p} \mathrm{~d} x: u \in W_{0}^{1, p}(\Omega), \int_{\Omega} g(x)|u|^{p} \mathrm{~d} x=1\right\} .
$$

See [7. Obviously, if $u$ is a minimizer, so is $|u|$; therefore we may assume $u \geq 0$.

The following lemma is taken from [6] and gives us the positivity of eigenfunctions associated to the principal eigenvalue.

Lemma 2.1 (6, Proposition 3.2). Let $g$ and $V$ be two measurable functions that satisfy the assumption (H1). If $u \in W_{0}^{1, p}(\Omega)$ is a nonnegative weak solution to (1.1), then either $u \equiv 0$ or $u>0$ for all $x \in \Omega$.

Proof. The proof is a direct consequence of Harnack's inequality. See [15].

We therefore immediately obtain

Corollary 2.2. Under the assumptions of Lemma 2.1, every eigenfunction associated to the principal positive eigenvalue has constant sign.

Furthermore, following [7, we have that the principal eigenvalue $\lambda(g, V)$ is simple.

Lemma 2.3. Let $g$ and $V$ be two measurable functions that satisfy the assumption (H1). Let $u$ and $v$ be two eigenfunctions associated to $\lambda(g, V)$. Then, there exists a constant $c \in \mathbb{R}$ such that $u=c v$.

Proof. The proof follows immediately from Lemma 4 in [7.

2.2. Results on rearrangements. We will now give some well-known results concerning the rearrangements of functions. They can be found, for instance, in [2, 3].

Definition 2.4. Given two measurable functions $f, g: \Omega \rightarrow \mathbb{R}$, we say that $f$ is a rearrangement of $g$ if

$$
|\{x \in \Omega: f(x) \geq \alpha\}|=|\{x \in \Omega: g(x) \geq \alpha\}| \quad \forall \alpha \in \mathbb{R},
$$

where $|\cdot|$ denotes the Lebesgue measure.

Now, given $f_{0} \in L^{p}(\Omega)$ the set of all rearrangements of $f_{0}$ is denoted by $\mathcal{R}\left(f_{0}\right)$, and $\overline{\mathcal{R}\left(f_{0}\right)}$ denotes the closure of $\mathcal{R}\left(f_{0}\right)$ in $L^{p}(\Omega)$ with respect to the weak topology.

Theorem 2.5. Let $1 \leq p<\infty$ and let $p^{\prime}$ be the conjugate exponent of $p$. Let $f_{0} \in L^{p}(\Omega), f_{0} \not \equiv 0$ and $g \in L^{p^{\prime}}(\Omega)$. Then, there exists $f_{*}, f^{*} \in \mathcal{R}\left(f_{0}\right)$ such that

$$
\int_{\Omega} f_{*} g \mathrm{~d} x \leq \int_{\Omega} f g \mathrm{~d} x \leq \int_{\Omega} f^{*} g \mathrm{~d} x \quad \forall f \in \overline{\mathcal{R}(f)} .
$$


Proof. The proof follows from Theorem 4 in [2].

Theorem 2.6. Let $1 \leq p \leq \infty$ and let $p^{\prime}$ be the conjugate of $p$. Let $f_{0} \in L^{p}(\Omega)$, $f_{0} \not \equiv 0$ and $g \in L^{p^{\prime}}(\Omega)$.

If the linear functional $L(f)=\int_{\Omega} f g \mathrm{~d} x$ has a unique maximizer $f^{*}$ relative to $\mathcal{R}\left(f_{0}\right)$, then there exists an increasing function $\phi$ such that $f^{*}=\phi \circ g$ a.e. in $\Omega$.

Furthermore, if the linear functional $L(f)$ has a unique minimizer $f_{*}$ relative to $\mathcal{R}\left(f_{0}\right)$, then there exists a decreasing function $\psi$ such that $f_{*}=\psi \circ g$ a.e. in $\Omega$.

Proof. The proof follows from Theorem 5 in [2].

\section{Minimization And CharaCterization}

Let $\Omega$ be a bounded smooth domain in $\mathbb{R}^{N}$ with $N \geq 2$ and $1<p<\infty$. Given measurable functions $g_{0}$ and $V_{0}$ that satisfy the assumptions (H1) and (H2), our aim in this section is to analyze the problem

$$
I=\inf \left\{\lambda(g, V): g \in \mathcal{R}\left(g_{0}\right), V \in \mathcal{R}\left(V_{0}\right)\right\},
$$

where $\mathcal{R}\left(g_{0}\right)$ (resp. $\mathcal{R}\left(V_{0}\right)$ ) is the set of all rearrangements of $g_{0}$ (resp. $V_{0}$ ) and $\lambda(g, V)$ is the first positive principal eigenvalue of problem (1.1).

Remark 3.1. Observe that if $g \in \mathcal{R}\left(g_{0}\right)$ and $V \in \mathcal{R}\left(V_{0}\right)$, then $g$ and $V$ satisfy (H1) and (H2).

We first need a lemma to show that, under hypotheses (H1) and (H2), the functionals

$$
J_{V}(u):=\int_{\Omega}|\nabla u|^{p} \mathrm{~d} x+\int_{\Omega} V(x)|u|^{p} \mathrm{~d} x
$$

are uniformly coercive for $V \in \mathcal{R}\left(V_{0}\right)$.

Lemma 3.2. Let $V_{0}$ satisfy (H1) and (H2). Then there exists $\delta_{0}>0$ such that

$$
J_{V}(u) \geq \delta_{0} \int_{\Omega}|\nabla u|^{p} \mathrm{~d} x, \quad \text { for every } V \in \mathcal{R}\left(V_{0}\right) .
$$

Proof. We prove the lemma, assuming that $\left\|V_{0}^{-}\right\|_{L^{q}(\Omega)}<S_{p q^{\prime}}$. Also, we assume that $1<p \leq N$. The other cases are easier and are left to the reader.

First, observe that

$$
J_{V}(u) \geq \int_{\Omega}|\nabla u|^{p} \mathrm{~d} x+\int_{\Omega} V^{-}(x)|u|^{p} \mathrm{~d} x .
$$

On the other hand, $q>N / p$ implies that $p q^{\prime}<p^{*}$. So

$$
\int_{\Omega}\left|V^{-}(x)\left\|\left.u\right|^{p} \mathrm{~d} x \leq\right\| V^{-}\left\|_{L^{q}(\Omega)}\right\| u\left\|_{L^{p q^{\prime}}(\Omega)}^{p}=\right\| V_{0}^{-}\left\|_{L^{q}(\Omega)}\right\| u \|_{L^{p q^{\prime}}(\Omega)}^{p} .\right.
$$

Then, by (H2), there exists $\delta_{0}$ such that

$$
\left\|V_{0}^{-}\right\|_{L^{q}(\Omega)} \leq\left(1-\delta_{0}\right) S_{p q^{\prime}} .
$$

Therefore

$$
J_{V}(u) \geq \delta_{0} \int_{\Omega}|\nabla u|^{p} \mathrm{~d} x
$$

as we wanted to prove. 
Remark 3.3. We remark that what is actually needed is the uniform coercitivity of the functionals $J_{V}$ for $V \in \mathcal{R}\left(V_{0}\right)$. Hypotheses (H1) and (H2) form a simple set of hypotheses that guarantee that.

We now prove that the infimum is achieved.

Theorem 3.4. Let $g_{0}$ and $V_{0}$ be measurable functions that satisfy the assumptions $(\mathrm{H} 1)$ and $(\mathrm{H} 2)$ and let $\mathcal{R}\left(g_{0}\right)$ and $\mathcal{R}\left(V_{0}\right)$ be the sets of all rearrangements of $g_{0}$ and $V_{0}$ respectively. Then there exist $g^{*} \in \mathcal{R}\left(g_{0}\right)$ and $V_{*} \in \mathcal{R}\left(V_{0}\right)$ such that

$$
I=\lambda\left(g^{*}, V_{*}\right) .
$$

Proof. Let $\left\{\left(g_{n}, V_{n}\right)\right\}_{n \in \mathbb{N}}$ be a minimizing sequence, i.e.,

$$
g_{n} \in \mathcal{R}\left(g_{0}\right) \text { and } V_{n} \in \mathcal{R}\left(V_{0}\right) \quad \forall n \in \mathbb{N}
$$

and

$$
I=\lim _{n \rightarrow \infty} \lambda\left(g_{n}, V_{n}\right)
$$

Let $u_{n}$ be the positive eigenfunction corresponding to $\lambda\left(g_{n}, V_{n}\right)$. Then

$$
\int_{\Omega} g_{n}(x) u_{n}^{p}=1 \quad \forall n \in \mathbb{N}
$$

and

$$
\lambda\left(g_{n}, V_{n}\right)=\int_{\Omega}\left|\nabla u_{n}\right|^{p}+V_{n}(x) u_{n}^{p} \mathrm{~d} x \quad \forall n \in \mathbb{N} .
$$

Hence

$$
I=\lim _{n \rightarrow \infty} \int_{\Omega}\left|\nabla u_{n}\right|^{p}+V_{n}(x) u_{n}^{p} \mathrm{~d} x .
$$

Thus, by Lemma 3.2. $\left\{u_{n}\right\}_{n \in \mathbb{N}}$ is bounded in $W_{0}^{1, p}(\Omega)$, and therefore there exists $u \in W_{0}^{1, p}(\Omega)$ and some subsequence of $\left\{u_{n}\right\}_{n \in \mathbb{N}}$ (still denoted by $\left\{u_{n}\right\}_{n \in \mathbb{N}}$ ) such that

$$
\begin{aligned}
& u_{n} \rightarrow u \text { weakly in } W^{1, p}(\Omega), \\
& u_{n} \rightarrow u \text { strongly in } L^{p q^{\prime}}(\Omega) .
\end{aligned}
$$

Recall that our assumptions on $q$ imply that $p q^{\prime}<p^{*}$.

On the other hand, $g_{n} \in \mathcal{R}\left(g_{0}\right)$ and $V_{n} \in \mathcal{R}\left(V_{0}\right)$ for all $n \in \mathbb{N}$. Then

$$
\left\|g_{n}\right\|_{L^{q}(\Omega)}=\left\|g_{0}\right\|_{L^{q}(\Omega)} \text { and }\left\|V_{n}\right\|_{L^{q}(\Omega)}=\left\|V_{0}\right\|_{L^{q}(\Omega)} \quad \forall n \in \mathbb{N} .
$$

Therefore there exist $f, W \in L^{q}(\Omega)$ and subsequences of $\left\{g_{n}\right\}_{n \in \mathbb{N}}$ and $\left\{V_{n}\right\}_{n \in \mathbb{N}}$ (still denoted by $\left\{g_{n}\right\}_{n \in \mathbb{N}}$ and $\left\{V_{n}\right\}_{n \in \mathbb{N}}$ ) such that

$$
\begin{aligned}
& g_{n} \rightarrow f \quad \text { weakly in } L^{q}(\Omega), \\
& V_{n} \rightarrow W \quad \text { weakly in } L^{q}(\Omega) .
\end{aligned}
$$

Thus, by (3.2), (3.3), (3.4) and (3.6), we have that

$$
I \geq \int_{\Omega}|\nabla u|^{p}+W(x)|u|^{p} \mathrm{~d} x
$$

and by (3.1), (3.4) and (3.5) we get

$$
\int_{\Omega} f(x)|u|^{p} \mathrm{~d} x=1
$$


Now, since $f \in \overline{\mathcal{R}\left(g_{0}\right)}$ and $W \in \overline{\mathcal{R}\left(V_{0}\right)}$, by Theorem 2.5, there exist $g^{*} \in \mathcal{R}\left(g_{0}\right)$ and $V_{*} \in \mathcal{R}\left(V_{0}\right)$ such that

$$
\alpha=\int_{\Omega} g^{*}(x)|u|^{p} \mathrm{~d} x \geq \int_{\Omega} f(x) u^{p} \mathrm{~d} x=1
$$

and

$$
\int_{\Omega} V_{*}(x) u^{p} \mathrm{~d} x \leq \int_{\Omega} W(x)|u|^{p} \mathrm{~d} x .
$$

Let $v=\alpha^{-1 / p}|u|$. Then

$$
\int_{\Omega} g^{*}(x) v^{p} \mathrm{~d} x=1
$$

and

$$
\int_{\Omega}|\nabla v|^{p}+V_{*}(x) v^{p} \mathrm{~d} x=\frac{1}{\alpha} \int_{\Omega}|\nabla u|^{p}+V_{*}(x)|u|^{p} \mathrm{~d} x \leq \frac{1}{\alpha} \int_{\Omega}|\nabla u|^{p}+W(x)|u|^{p} \mathrm{~d} x .
$$

Consequently

$$
\lambda\left(g^{*}, V_{*}\right) \leq I .
$$

Then

$$
I=\lambda\left(g^{*}, V_{*}\right) .
$$

The proof is now complete.

Now we give a characterization of $g^{*}$ and $V_{*}$.

Theorem 3.5. Let $g_{0}$ and $V_{0}$ be measurable functions that satisfy the assumptions $(\mathrm{H} 1)$ and $(\mathrm{H} 2)$. Let $g^{*} \in \mathcal{R}\left(g_{0}\right)$ and $V_{*} \in \mathcal{R}\left(V_{0}\right)$ be such that $\lambda\left(g^{*}, V_{*}\right)=I$ are the measurable functions given by Theorem 3.4. Then there exist an increasing function $\phi$ and a decreasing function $\psi$ such that

$$
\begin{gathered}
g^{*}=\phi\left(u_{*}\right) \quad \text { a.e. in } \Omega, \\
V_{*}=\psi\left(u_{*}\right) \quad \text { a.e. in } \Omega,
\end{gathered}
$$

where $u_{*}$ is the positive eigenfunction associated to $\lambda\left(g^{*}, V_{*}\right)$.

Proof. We proceed in four steps.

Step 1. First we show that $V_{*}$ is a minimizer of the linear functional

$$
L(V):=\int_{\Omega} V(x) u_{*}^{p} \mathrm{~d} x
$$

relative to $V \in \overline{\mathcal{R}\left(V_{0}\right)}$.

We have that

$$
\int_{\Omega} g^{*}(x) u_{*}^{p} \mathrm{~d} x=1
$$

and

$$
I=\lambda\left(g^{*}, V_{*}\right)=\int_{\Omega}\left|\nabla u_{*}\right|^{p}+V_{*}(x) u_{*}^{p} \mathrm{~d} x .
$$

Then, for all $V \in \mathcal{R}\left(V_{0}\right)$,

$$
\int_{\Omega}\left|\nabla u_{*}\right|^{p}+V_{*}(x) u_{*}^{p} \mathrm{~d} x \leq \lambda\left(g^{*}, V\right) \leq \int_{\Omega}\left|\nabla u_{*}\right|^{p}+V(x) u_{*}^{p} \mathrm{~d} x
$$

and therefore

$$
\int_{\Omega} V_{*}(x) u_{*}^{p} \mathrm{~d} x \leq \int_{\Omega} V(x) u_{*}^{p} \mathrm{~d} x \quad \forall V \in \mathcal{R}\left(V_{0}\right) .
$$


Thus, we can conclude that

$$
\int_{\Omega} V_{*}(x) u_{*}^{p} \mathrm{~d} x=\inf \left\{L(V): V \in \overline{\mathcal{R}\left(V_{0}\right)}\right\} .
$$

Step 2. We show that $V_{*}$ is the unique minimizer of $L(V)$ relative to $\mathcal{R}\left(V_{0}\right)$.

Suppose that $W$ is another minimizer of $L(V)$ relative to $\mathcal{R}\left(V_{0}\right)$. Then

$$
\int_{\Omega} V_{*}(x) u_{*}^{p} \mathrm{~d} x=\int_{\Omega} W(x) u_{*}^{p} \mathrm{~d} x .
$$

Thus

$$
\begin{aligned}
I & =\lambda\left(g^{*}, V_{*}\right) \\
& =\int_{\Omega}\left|\nabla u_{*}\right|^{p}+V_{*}(x) u_{*}^{p} \mathrm{~d} x \\
& =\int_{\Omega}\left|\nabla u_{*}\right|^{p}+W(x) u_{*}^{p} \mathrm{~d} x \\
& \geq \lambda\left(g^{*}, W\right) \\
& \geq I .
\end{aligned}
$$

Hence $u_{*}$ is the positive eigenfunction associated to $\lambda\left(g^{*}, V_{*}\right)=\lambda\left(g^{*}, W\right)$. Then

$$
\begin{array}{lll}
-\Delta_{p} u_{*}+V_{*}(x) u_{*}^{p-1} & =\lambda\left(g^{*}, V_{*}\right) g^{*}(x) u^{p-1} & \text { in } \Omega, \\
-\Delta_{p} u_{*}+W(x) u_{*}^{p-1} & =\lambda\left(g^{*}, V_{*}\right) g^{*}(x) u^{p-1} & \text { in } \Omega .
\end{array}
$$

Subtracting (3.8) from (3.7), we get

$$
\left(V_{*}(x)-W(x)\right) u_{*}^{p-1}=0 \quad \text { a.e. in } \Omega .
$$

Then $V_{*}=W$ a.e. in $\Omega$.

Thus, by Theorem 2.6, there exists a decreasing function $\psi$ such that

$$
V_{*}=\psi\left(u_{*}\right) \quad \text { a.e. in } \Omega .
$$

Step 3. Now, we show that $g^{*}$ is a maximizer of the linear functional

$$
H(g):=\int_{\Omega} g(x) u_{*}^{p} \mathrm{~d} x
$$

relative to $g \in \overline{\mathcal{R}\left(g_{0}\right)}$.

We argue by contradiction, so assume that there exists $g \in \mathcal{R}\left(g_{0}\right)$ such that

$$
\alpha=\int_{\Omega} g(x) u_{*}^{p} \mathrm{~d} x>\int_{\Omega} g^{*}(x) u_{*}^{p} \mathrm{~d} x=1
$$

and take $v=\alpha^{-1 / p} u_{*}$. Then

$$
\int_{\Omega} g(x) v^{p} \mathrm{~d} x=1
$$

and

$$
\int_{\Omega}|\nabla v|^{p}+V_{*}(x) v^{p} \mathrm{~d} x=\frac{1}{\alpha} \int_{\Omega}\left|\nabla u_{*}\right|^{p}+V_{*}(x) u_{*}^{p} \mathrm{~d} x=\frac{1}{\alpha} \lambda\left(g^{*}, V_{*}\right)<\lambda\left(g^{*}, V_{*}\right) .
$$

Therefore

$$
\lambda\left(g, V_{*}\right)<\lambda\left(g^{*}, V_{*}\right),
$$

which contradicts the minimality of $\lambda\left(g^{*}, V_{*}\right)$.

Step 4. Finally, we show that $g^{*}$ is the unique maximizer of $H(g)$ relative to $\mathcal{R}\left(g_{0}\right)$. 
Assume that there exists another maximizer $f$ of $H(g)$ relative to $\mathcal{R}\left(g_{0}\right)$. Then

$$
\int_{\Omega} f(x) u_{*}^{p} \mathrm{~d} x=\int_{\Omega} g^{*}(x) u_{*}^{p} \mathrm{~d} x=1
$$

and therefore

$$
I=\lambda\left(g^{*}, V_{*}\right) \leq \lambda\left(f, V_{*}\right) \leq \int_{\Omega}\left|\nabla u_{*}\right|^{p}+V_{*}(x) u_{*}^{p} \mathrm{~d} x=I .
$$

Then $\lambda\left(g^{*}, V_{*}\right)=\lambda\left(f, V_{*}\right)$, and hence $u_{*}$ is the eigenfunction associated to $\lambda\left(g^{*}, V_{*}\right)$ $=\lambda\left(f, V_{*}\right)$. Thus

$$
\begin{array}{rll}
-\Delta_{p} u_{*}+V_{*}(x) u_{*}^{p-1} & =\lambda\left(g^{*}, V_{*}\right) g^{*}(x) u_{*}^{p-1} & \text { in } \Omega, \\
-\Delta_{p} u_{*}+V_{*}(x) u_{*}^{p-1} & =\lambda\left(g^{*}, V_{*}\right) f(x) u_{*}^{p-1} & \text { in } \Omega .
\end{array}
$$

Subtracting (3.10) from (3.9), we get

$$
\lambda\left(g^{*}, V_{*}\right)\left(g^{*}(x)-f(x)\right) u_{*}^{p}=0 \quad \text { a.e. in } \Omega ;
$$

thus $g^{*}=f$ a.e. in $\Omega$.

Then, by Theorem 2.6. there exists an increasing function $\phi$ such that

$$
g^{*}=\phi\left(u_{*}\right) \quad \text { a.e. in } \Omega .
$$

This finishes the proof.

\section{Differentiation of $\lambda(g, V)$}

The first aim of this section is to prove the continuity of the first positive eigenvalue $\lambda(g, V)$ with respect to $g$ and $V$. Then we proceed further and compute the derivative of $\lambda(g, V)$ with respect to perturbations in $g$ and $V$.

Proposition 4.1. The first positive eigenvalue $\lambda(g, V)$ of (1.1) is continuous with respect to $(g, V) \in \mathcal{A}$, where

$$
\mathcal{A}:=\left\{(g, V) \in L^{q}(\Omega) \times L^{q}(\Omega):(g, V) \text { satisfies }(\mathrm{H} 1) \text { and }(\mathrm{H} 2)\right\} ;
$$

i.e.,

$$
\lambda\left(g_{n}, V_{n}\right) \rightarrow \lambda(g, V)
$$

when $\left(g_{n}, V_{n}\right) \rightarrow(g, V)$ strongly in $L^{q}(\Omega) \times L^{q}(\Omega)$ and $\left(g_{n}, V_{n}\right),(g, V) \in \mathcal{A}$.

Proof. We know that

$$
\lambda\left(g_{n}, V_{n}\right)=\int_{\Omega}\left|\nabla u_{n}\right|^{p}+V_{n}(x) u_{n}^{p} \mathrm{~d} x
$$

and

with

$$
\lambda(g, V)=\int_{\Omega}|\nabla u|^{p}+V(x) u^{p} \mathrm{~d} x
$$

$$
\int_{\Omega} g_{n}(x) u_{n}^{p} \mathrm{~d} x=\int_{\Omega} g(x) u^{p} \mathrm{~d} x=1,
$$

where $u_{n}$ and $u$ are the positive eigenfunctions associated to $\lambda\left(g_{n}, V_{n}\right)$ and $\lambda(g, V)$ respectively.

We begin by observing that

$$
H\left(g_{n}\right):=\int_{\Omega} g_{n}(x) u^{p} \mathrm{~d} x=\int_{\Omega}\left(g_{n}(x)-g(x)\right) u^{p} \mathrm{~d} x+1 \rightarrow 1,
$$


as $n \rightarrow \infty$. Then there exists $n_{0} \in \mathbb{N}$ such that

$$
H\left(g_{n}\right)>0 \quad \forall n \geq n_{0} .
$$

Thus we take $v_{n}:=H\left(g_{n}\right)^{-1 / p} u$, and by (2.1) we have

$$
\lambda\left(g_{n}, V_{n}\right) \leq \int_{\Omega}\left|\nabla v_{n}\right|^{p}+V_{n}(x) v_{n}^{p} \mathrm{~d} x=\frac{1}{H\left(g_{n}\right)} \int_{\Omega}|\nabla u|^{p}+V_{n}(x) u^{p} \mathrm{~d} x .
$$

Therefore, taking limits when $g_{n} \rightarrow g$ and $V_{n} \rightarrow V$ in $L^{q}(\Omega)$, we get that

$$
\limsup _{n \rightarrow \infty} \lambda\left(g_{n}, V_{n}\right) \leq \int_{\Omega}|\nabla u|^{p}+V(x) u^{p} \mathrm{~d} x=\lambda(g, V) .
$$

On the other hand, as $V_{n} \rightarrow V$ strongly in $L^{q}(\Omega)$, it is easy to see that there exist $n_{1} \in \mathbb{N}$ and $\delta_{1}>0$ such that

$$
\left\|V_{n}^{-}\right\|_{L^{q}(\Omega)},\left\|V^{-}\right\|_{L^{q}(\Omega)}<S_{p q^{\prime}}\left(1-\delta_{1}\right) \quad \forall n \geq n_{1},
$$

or there exist a subsequence of $\left\{V_{n}\right\}_{n \in \mathbb{N}}$, which we denote again by $\left\{V_{n}\right\}_{n \in \mathbb{N}}$, and $\delta_{2}>0$ such that

$$
V_{n}, V>-S_{p}+\delta_{2} \quad \forall n \in \mathbb{N} .
$$

Therefore, as $\left\{\lambda\left(g_{n}, V_{n}\right)\right\}_{n \in \mathbb{N}}$ is bounded, arguing as in Lemma 3.2 we have that $\left\{u_{n}\right\}_{n \in \mathbb{N}}$ is bounded in $W_{0}^{1, p}(\Omega)$. Therefore there exist $v \in W_{0}^{1, p}(\Omega)$ and a subsequence of $\left\{u_{n}\right\}_{n \in \mathbb{N}}$ (that we still denote by $\left\{u_{n}\right\}_{n \in \mathbb{N}}$ ) such that

$$
\begin{aligned}
& u_{n} \rightarrow v \quad \text { weakly in } W_{0}^{1, p}(\Omega), \\
& u_{n} \rightarrow v \quad \text { strongly in } L^{p q^{\prime}}(\Omega) .
\end{aligned}
$$

By (4.2) and as $g_{n} \rightarrow g$ in $L^{q}(\Omega)$ we have that

$$
1=\lim _{n \rightarrow \infty} \int_{\Omega} g_{n}(x)\left|u_{n}\right|^{p} \mathrm{~d} x=\int_{\Omega} g(x)|v|^{p} \mathrm{~d} x .
$$

Finally, by (4.1), (4.2) and, as $V_{n} \rightarrow V$ in $L^{q}(\Omega)$, we arrive at

$$
\begin{aligned}
\liminf _{n \rightarrow \infty} \lambda\left(g_{n}, V_{n}\right) & =\liminf _{n \rightarrow \infty} \int_{\Omega}\left|\nabla u_{n}\right|^{p}+V_{n}(x) u_{n}^{p} \mathrm{~d} x \\
& \geq \int_{\Omega}|\nabla v|^{p}+V(x)|v|^{p} \mathrm{~d} x \\
& \geq \lambda(g, V)
\end{aligned}
$$

and the result follows.

Remark 4.2. Observe that if instead of (H2) we required only that $V>-S_{p}+\delta$, the exact same proof of Proposition 4.1 gives the continuity of $\lambda(g, V)$ with respect to weak convergence.

Now we arrive at the main result of this section; namely, we compute the derivative of the first positive eigenvalue $\lambda(g, V)$ with respect to perturbations in $g$ and $V$.

We begin by describing the kind of variations that we are going to consider. Let $W$ be a regular (smooth) vector field, globally Lipschitz, with support in $\Omega$ and let $\varphi_{t}: \mathbb{R}^{N} \rightarrow \mathbb{R}^{N}$ be the flow defined by

$$
\begin{cases}\frac{\mathrm{d}}{\mathrm{d} t} \varphi_{t}(x)=W\left(\varphi_{t}(x)\right) & t>0, \\ \varphi_{0}(x)=x & x \in \mathbb{R}^{N} .\end{cases}
$$


We have

$$
\varphi_{t}(x)=x+t W(x)+o(t) \quad \forall x \in \mathbb{R}^{N} .
$$

Thus, if $g$ and $V$ are measurable functions that satisfy the assumptions (H1) and (H2), we define $g_{t}:=g \circ \varphi_{t}^{-1}$ and $V_{t}:=V \circ \varphi_{t}^{-1}$. Now, let

$$
\lambda(t):=\lambda\left(g_{t}, V_{t}\right)=\int_{\Omega}\left|\nabla u_{t}\right|^{p}+V_{t}(x)\left|u_{t}\right|^{p} \mathrm{~d} x,
$$

with

$$
\int_{\Omega} g_{t}(x) u_{t}^{p} \mathrm{~d} x=1
$$

where $u_{t}$ is the eigenfunction associated to $\lambda(t)$.

Remark 4.3. In order for this approach to be useful for the optimization problem of the previous section, we need to guarantee that $g_{t} \in \mathcal{R}\left(g_{0}\right)$ and $V_{t} \in \mathcal{R}\left(V_{0}\right)$ whenever $g \in \mathcal{R}\left(g_{0}\right)$ and $V \in \mathcal{R}\left(V_{0}\right)$.

It is not difficult to check that this is true for incompressible deformation fields, i.e., for those $W$ 's such that

$$
\operatorname{div} W=0 .
$$

Lemma 4.4. Given $f \in L^{q}(\Omega)$, then

$$
f_{t}:=f \circ \varphi_{t}^{-1} \rightarrow f \text { in } L^{q}(\Omega) \text {, as } t \rightarrow 0^{+} .
$$

Proof. Let $\varepsilon>0$ and let $g \in C_{c}^{\infty}(\Omega)$ be fixed such that $\|f-g\|_{L^{q}(\Omega)}<\varepsilon$. By the usual change of variables formula, we have

$$
\left\|f_{t}-g_{t}\right\|_{L^{q}(\Omega)}^{q}=\int_{\Omega}|f-g|^{q} J \varphi_{t} \mathrm{~d} x
$$

where $g_{t}=g \circ \varphi_{t}^{-1}$ and $J \varphi_{t}$ is the Jacobian of $\varphi_{t}$. We know that

$$
J \varphi_{t}=1+t \operatorname{div} W+o(t) .
$$

Here $\operatorname{div} W$ is the divergence of $W$. Then

$$
\left\|f_{t}-g_{t}\right\|_{L^{q}(\Omega)}^{q}=\int_{\Omega}|f-g|^{q}(1+t \operatorname{div} W+o(t)) \mathrm{d} x .
$$

Then, there exist $t_{1}>0$ such that if $0<t<t_{1}$, then

$$
\left\|f_{t}-g_{t}\right\|_{L^{q}(\Omega)}<C \varepsilon
$$

where $C$ is a constant independent of $t$. Moreover, since $\varphi_{t}^{-1} \rightarrow I d$ in the $C^{1}$ topology when $t \rightarrow 0$, then $g_{t}=g \circ \varphi_{t}^{-1} \rightarrow g$ in the $C^{1}$ topology and therefore there exist $t_{2}>0$ such that if $0<t<t_{2}$, then

$$
\left\|g_{t}-g\right\|_{L^{q}(\Omega)}<\varepsilon .
$$

Finally, we have for all $0<t<t_{0}=\min \left\{t_{1}, t_{2}\right\}$ that

$$
\left\|f_{t}-f\right\|_{L^{q}(\Omega)} \leq\left\|f_{t}-g_{t}\right\|_{L^{q}(\Omega)}+\left\|g_{t}-g\right\|_{L^{q}(\Omega)}+\|f-g\|_{L^{q}(\Omega)} \leq C \varepsilon,
$$

where $C$ is a constant independent of $t$.

By Proposition 4.1 and Lemma 4.4 we have that

Corollary 4.5. Let $g$ and $V$ be measurable functions that satisfy the assumptions $(\mathrm{H} 1)$ and $(\mathrm{H} 2)$. Then, with the previous notation, $\lambda(t)$ is continuous at $t=0$, i.e.,

$$
\lambda(t) \rightarrow \lambda(0)=\lambda(g, V) \quad \text { as } t \rightarrow 0^{+} .
$$


Lemma 4.6. Let $g$ and $V$ be measurable functions that satisfy the assumptions $(\mathrm{H} 1)$ and $(\mathrm{H} 2)$. Let $u_{t}$ be the normalized positive eigenfunction associated to $\lambda(t)$ with $t>0$. Then

$$
\lim _{t \rightarrow 0^{+}} u_{t}=u_{0} \quad \text { strongly in } W_{0}^{1, p}(\Omega),
$$

where $u_{0}$ is the unique normalized positive eigenfunction associated to $\lambda(g, V)$.

Proof. From the previous corollary we deduce that $\lambda(t)$ is bounded and, as in the proof of Proposition 4.1, we further deduce that $\left\{u_{t}\right\}$ is bounded in $W_{0}^{1, p}(\Omega)$.

So, given $\left\{t_{n}\right\}_{n \in \mathbb{N}}$, we have that $\left\{u_{t_{n}}\right\}_{n \in \mathbb{N}}$ is bounded in $W_{0}^{1, p}(\Omega)$, and therefore there exist $u_{0} \in W_{0}^{1, p}(\Omega)$ and some subsequence (still denoted by $\left\{u_{t_{n}}\right\}_{n \in \mathbb{N}}$ ) such that

$$
\begin{aligned}
& u_{t_{n}} \rightarrow u_{0} \quad \text { weakly in } W_{0}^{1, p}(\Omega), \\
& u_{t_{n}} \rightarrow u_{0} \quad \text { strongly in } L^{p q^{\prime}}(\Omega) .
\end{aligned}
$$

Since $\left(g_{t_{n}}, V_{t_{n}}\right) \rightarrow(g, V)$ strongly in $L^{q}(\Omega) \times L^{q}(\Omega)$ as $n \rightarrow \infty$ and by (4.5), we get

$$
1=\lim _{n \rightarrow \infty} \int_{\Omega} g_{t_{n}}(x)\left|u_{t_{n}}\right|^{p} \mathrm{~d} x=\int_{\Omega} g(x)\left|u_{0}\right|^{p} \mathrm{~d} x
$$

and

$$
\lim _{n \rightarrow \infty} \int_{\Omega} V_{t_{n}}(x)\left|u_{t_{n}}\right|^{p} \mathrm{~d} x=\int_{\Omega} V(x)\left|u_{0}\right|^{p} \mathrm{~d} x .
$$

Thus, using (4.4),

$$
\begin{aligned}
\lambda(0) & =\lim _{n \rightarrow \infty} \lambda\left(t_{n}\right) \\
& =\lim _{n \rightarrow \infty} \int_{\Omega}\left|\nabla u_{t_{n}}\right|^{p}+V_{t_{n}}(x)\left|u_{t_{n}}\right|^{p} \mathrm{~d} x \\
& \geq \int_{\Omega}\left|\nabla u_{0}\right|^{p}+V(x)\left|u_{0}\right|^{p} \mathrm{~d} x \\
& \geq \lambda(0) .
\end{aligned}
$$

Then $u_{0}$ is the normalized eigenfunction associated to $\lambda(0)$, and, as $\left\{u_{t_{n}}\right\}_{n \in \mathbb{N}}$ are positive, it follows that $u_{0}$ is positive.

Moreover

$$
\left\|\nabla u_{t_{n}}\right\|_{L^{p}(\Omega)} \rightarrow\left\|\nabla u_{0}\right\|_{L^{p}(\Omega)} \quad \text { as } n \rightarrow \infty .
$$

Then, again using (4.4), we have

$$
u_{t_{n}} \rightarrow u_{0} \quad \text { in } W_{0}^{1, p}(\Omega) \text { as } n \rightarrow \infty,
$$

as we wanted to show.

Remark 4.7. It is easy to see that, as $\varphi_{t} \rightarrow I d$ in the $C^{1}$ topology, it follows from Lemma 4.6 that

$$
u_{t} \circ \varphi_{t} \rightarrow u_{0} \quad \text { strongly in } W_{0}^{1, p}(\Omega) \text { as } t \rightarrow 0,
$$

when $u_{t} \rightarrow u_{0}$ strongly in $W_{0}^{1, p}(\Omega)$.

Now, we arrive at the main result of this section. 
Theorem 4.8. With the previous notation, if $g$ and $V$ are measurable functions that satisfy the assumptions $(\mathrm{H} 1)$ and $(\mathrm{H} 2)$, we have that $\lambda(t)$ is differentiable at $t=0$ and

$$
\begin{aligned}
\left.\frac{\mathrm{d} \lambda(t)}{\mathrm{d} t}\right|_{t=0}= & \int_{\Omega}\left(\left|\nabla u_{0}\right|^{p}+V(x)\left|u_{0}\right|^{p}\right) \operatorname{div} W \mathrm{~d} x-p \int_{\Omega}\left|\nabla u_{0}\right|^{p-2}\left\langle\nabla u_{0},{ }^{T} W^{\prime} \nabla u_{0}^{T}\right\rangle \mathrm{d} x \\
& -\lambda(0) \int_{\Omega} g(x)\left|u_{0}\right|^{p} \operatorname{div} W \mathrm{~d} x,
\end{aligned}
$$

where $W^{\prime}$ denotes the differential matrix of $W,{ }^{T} A$ is the transpose of the matrix $A, x^{T}$ is the transpose of the vector $x$ and $u_{0}$ is the eigenfunction associated to $\lambda(0)=\lambda(g, V)$.

Proof. First we consider $v_{t}:=u_{0} \circ \varphi_{t}^{-1}$. Then, by the change of variables formula we get

$$
\begin{aligned}
\int_{\Omega} g_{t}(x)\left|v_{t}\right|^{p} \mathrm{~d} x & =\int_{\Omega} g(x)\left|u_{0}\right|^{p} J \varphi_{t} \mathrm{~d} x \\
& =\int_{\Omega} g(x)\left|u_{0}\right|^{p}(1+t \operatorname{div} W+o(t)) \mathrm{d} x \\
& =1+t \int_{\Omega} g(x)\left|u_{0}\right|^{p} \operatorname{div} W \mathrm{~d} x+o(t), \\
\int_{\Omega} V_{t}(x)\left|v_{t}\right|^{p} \mathrm{~d} x= & \int_{\Omega} V(x)\left|u_{0}\right|^{p} J \varphi_{t} \mathrm{~d} x \\
& =\int_{\Omega} V(x)\left|u_{0}\right|^{p}(1+t \operatorname{div} W+o(t)) \mathrm{d} x \\
& =\int_{\Omega} V(x)\left|u_{0}\right|^{p} \mathrm{~d} x+t \int_{\Omega} V(x)\left|u_{0}\right|^{p} \operatorname{div} W \mathrm{~d} x+o(t), \\
\int_{\Omega}\left|\nabla v_{t}\right|^{p} \mathrm{~d} x= & \int_{\Omega}\left|{ }^{T}\left[\varphi_{t}^{\prime}\right]^{-1}(x) \nabla u_{0}^{T}\right|^{p} J \varphi_{t} \mathrm{~d} x \\
= & \int_{\Omega}\left|\left(I-t^{T} W^{\prime}+o(t)\right) \nabla u_{0}^{T}\right|^{p}(1+t \operatorname{div} W+o(t)) \mathrm{d} x \\
= & \int_{\Omega}\left(\left|\nabla u_{0}\right|^{p}-t p\left|\nabla u_{0}\right|^{p-2}\left\langle\nabla u_{0},{ }^{T} W^{\prime} \nabla u_{0}^{T}\right\rangle\right)(1+t \operatorname{div} W) \mathrm{d} x+o(t) \\
= & \int_{\Omega}\left|\nabla u_{0}\right|^{p} \mathrm{~d} x+t \int_{\Omega}\left|\nabla u_{0}\right|^{p} \operatorname{div} W \mathrm{~d} x \\
& -t p \int_{\Omega}\left|\nabla u_{0}\right|^{p-2}\left\langle\nabla u_{0},{ }^{T} W^{\prime} \nabla u_{0}^{T}\right\rangle \mathrm{d} x+o(t) .
\end{aligned}
$$

Then, for $t$ small enough,

$$
\int_{\Omega} g_{t}(x)\left|v_{t}\right|^{p} \mathrm{~d} x>0
$$

and therefore

$$
\lambda(t) \leq \frac{\int_{\Omega}\left|\nabla v_{t}\right|^{p}+V_{t}(x)\left|v_{t}\right|^{p} \mathrm{~d} x}{\int_{\Omega} g_{t}(x)\left|v_{t}\right|^{p} \mathrm{~d} x}
$$

So

$$
\lambda(t) \int_{\Omega} g_{t}(x)\left|v_{t}\right|^{p} \mathrm{~d} x \leq \int_{\Omega}\left|\nabla v_{t}\right|^{p}+V_{t}(x)\left|v_{t}\right|^{p} \mathrm{~d} x .
$$


Then we have that

$$
\begin{aligned}
\lambda(t)\left(1+t \int_{\Omega} g(x)\left|u_{0}\right|^{p} \operatorname{div} W \mathrm{~d} x\right) \leq & \int_{\Omega}\left|\nabla u_{0}\right|^{p}+V(x)\left|u_{0}\right|^{p} \mathrm{~d} x \\
& +t \int_{\Omega}\left(\left|\nabla u_{0}\right|^{p}+V(x)\left|u_{0}\right|^{p}\right) \operatorname{div} W \mathrm{~d} x \\
& -t p \int_{\Omega}\left|\nabla u_{0}\right|^{p-2}\left\langle\nabla u_{0},{ }^{T} W^{\prime} \nabla u_{0}^{T}\right\rangle \mathrm{d} x+o(t) \\
= & \lambda(0)+t \int_{\Omega}\left(\left|\nabla u_{0}\right|^{p}+V(x)\left|u_{0}\right|^{p}\right) \operatorname{div} W \mathrm{~d} x \\
& -t p \int_{\Omega}\left|\nabla u_{0}\right|^{p-2}\left\langle\nabla u_{0},{ }^{T} W^{\prime} \nabla u_{0}^{T}\right\rangle \mathrm{d} x+o(t),
\end{aligned}
$$

and we get that

$$
\begin{aligned}
\frac{\lambda(t)-\lambda(0)}{t} \leq & \int_{\Omega}\left(\left|\nabla u_{0}\right|^{p}+V(x)\left|u_{0}\right|^{p}\right) \operatorname{div} W \mathrm{~d} x \\
& -p \int_{\Omega}\left|\nabla u_{0}\right|^{p-2}\left\langle\nabla u_{0}{ }^{T} W^{\prime} \nabla u_{0}^{T}\right\rangle \mathrm{d} x \\
& -\lambda(t) \int_{\Omega} g(x)\left|u_{0}\right|^{p} \operatorname{div} W \mathrm{~d} x+o(1) .
\end{aligned}
$$

In a similar way, if we take $w_{t}=u_{t} \circ \varphi_{t}$ we have that

$$
\begin{aligned}
\frac{\lambda(t)-\lambda(0)}{t} \geq & \int_{\Omega}\left(\left|\nabla w_{t}\right|^{p}+V(x)\left|w_{t}\right|^{p}\right) \operatorname{div} W \mathrm{~d} x \\
& -p \int_{\Omega}\left|\nabla w_{t}\right|^{p-2}\left\langle\nabla w_{t},{ }^{T} W^{\prime} \nabla w_{t}^{T}\right\rangle \mathrm{d} x \\
& -\lambda(0) \int_{\Omega} g(x)\left|w_{t}\right|^{p} \operatorname{div} W \mathrm{~d} x+o(1) .
\end{aligned}
$$

Thus, taking the limit in the two last inequalities as $t \rightarrow 0^{+}$, by Corollary 4.5 and Remark 4.7, we get that

$$
\begin{aligned}
\lim _{t \rightarrow 0^{+}} \frac{\lambda(t)-\lambda(0)}{t}= & \int_{\Omega}\left(\left|\nabla u_{0}\right|^{p}+V(x)\left|u_{0}\right|^{p}\right) \operatorname{div} W \mathrm{~d} x \\
& -p \int_{\Omega}\left|\nabla u_{0}\right|^{p-2}\left\langle\nabla u_{0},{ }^{T} W^{\prime} \nabla u_{0}^{T}\right\rangle \mathrm{d} x \\
& -\lambda(0) \int_{\Omega} g(x)\left|u_{0}\right|^{p} \operatorname{div} W \mathrm{~d} x .
\end{aligned}
$$

This finishes the proof.

Remark 4.9. When we work in the class of rearrangements of a fixed pair $\left(g_{0}, V_{0}\right)$, as was mentioned in Remark 4.3. we need the deformation field $W$ to verify that $\operatorname{div} W=0$. So, in this case, the formula for $\lambda^{\prime}(0)$ reads

$$
\left.\frac{\mathrm{d} \lambda(t)}{\mathrm{d} t}\right|_{t=0}=-p \int_{\Omega}\left|\nabla u_{0}\right|^{p-2}\left\langle\nabla u_{0},{ }^{T} W^{\prime} \nabla u_{0}^{T}\right\rangle \mathrm{d} x .
$$

In order to improve the expression for the formula of $\lambda^{\prime}(0)$ we need a lemma that will allow us to regularize problem (1.1) since solutions to (1.1) are $C^{1, \delta}$ for some $\delta>0$ but are not $C^{2}$ nor $W^{2, q}$ in general (see [16]). 
Lemma 4.10. Let $V, g$ be measurable functions that satisfy the assumptions (H1) and (H2) and let $V_{\varepsilon}, g_{\varepsilon} \in C_{0}^{\infty}(\Omega)$ be such that $V_{\varepsilon} \rightarrow V$ and $g_{\varepsilon} \rightarrow g$ in $L^{q}(\Omega)$. Let

$$
\lambda_{\varepsilon}:=\min _{\substack{v \in W_{0}^{1, p},(\Omega) \\ \int_{\Omega} g_{\varepsilon}(x)|v|^{p} \mathrm{~d} x=1}} \int_{\Omega}\left(|\nabla v|^{2}+\varepsilon^{2}\right)^{(p-2) / 2}|\nabla v|^{2}+V_{\varepsilon}(x)|v|^{p} \mathrm{~d} x .
$$

Finally, let $u_{\varepsilon}$ be the unique normalized positive eigenfunction associated to $\lambda_{\varepsilon}$.

Then, $\lambda_{\varepsilon} \rightarrow \lambda(g, V)$ and $u_{\varepsilon} \rightarrow u_{0}$ strongly in $W_{0}^{1, p}(\Omega)$, where $u_{0}$ is the unique normalized positive eigenfunction associated to $\lambda(g, V)$.

Proof. First, observe that, as $g_{\varepsilon} \rightarrow g$ in $L^{q}(\Omega)$, if $u_{0}$ is the normalized positive eigenfunction associated to $\lambda(g, V)$, we have that

$$
\int_{\Omega} g_{\varepsilon}(x)\left|u_{0}\right|^{p} \mathrm{~d} x>0
$$

for all $\varepsilon>0$ small enough. Then, for all $\varepsilon>0$ small enough, taking

$$
v_{\varepsilon}=\frac{u_{0}}{\left(\int_{\Omega} g_{\varepsilon}(x)\left|u_{0}\right|^{p} \mathrm{~d} x\right)^{1 / p}}
$$

in the characterization of $\lambda_{\varepsilon}$, we get

$$
\lambda_{\varepsilon} \leq \int_{\Omega}\left(\left|\nabla v_{\varepsilon}\right|^{2}+\varepsilon^{2}\right)^{(p-2) / 2}\left|\nabla v_{\varepsilon}\right|^{2}+V_{\varepsilon}(x)\left|v_{\varepsilon}\right|^{p} \mathrm{~d} x .
$$

Hence, passing to the limit as $\varepsilon \rightarrow 0^{+}$, since $\int_{\Omega} g_{\varepsilon}(x)\left|u_{0}\right|^{p} \mathrm{~d} x \rightarrow \int_{\Omega} g(x)\left|u_{0}\right|^{p} \mathrm{~d} x=1$ as $\varepsilon \rightarrow 0^{+}$, we arrive at

$$
\limsup _{\varepsilon \rightarrow 0^{+}} \lambda_{\varepsilon} \leq \lambda(g, V) .
$$

Now, for any $v \in W_{0}^{1, p}(\Omega)$ normalized such that

$$
\int_{\Omega} g_{\varepsilon}(x)|v|^{p} \mathrm{~d} x=1
$$

we have that

$$
\int_{\Omega}\left(|\nabla v|^{2}+\varepsilon^{2}\right)^{(p-2) / 2}|\nabla v|^{2}+V_{\varepsilon}(x)|v|^{p} \mathrm{~d} x \geq \int_{\Omega}|\nabla v|^{p}+V_{\varepsilon}(x)|v|^{p} \mathrm{~d} x \geq \lambda\left(g_{\varepsilon}, V_{\varepsilon}\right) ;
$$

therefore $\lambda_{\varepsilon} \geq \lambda\left(g_{\varepsilon}, V_{\varepsilon}\right)$.

Now, by Proposition 4.1, we have that $\lambda\left(g_{\varepsilon}, V_{\varepsilon}\right) \rightarrow \lambda(g, V)$ as $\varepsilon \rightarrow 0^{+}$. So

$$
\liminf _{\varepsilon \rightarrow 0^{+}} \lambda_{\varepsilon} \geq \lambda(g, V) .
$$

Finally, from the convergence of the eigenvalues, it is easy to see that the normalized eigenfunctions $u_{\varepsilon}$ associated to $\lambda_{\varepsilon}$ are bounded in $W_{0}^{1, p}(\Omega)$ uniformly in $\varepsilon>0$. Therefore, there exist a sequence, which we still call $\left\{u_{\varepsilon}\right\}$, and a function $u \in W_{0}^{1, p}(\Omega)$ such that

$$
\begin{array}{ll}
u_{\varepsilon} \rightarrow u & \text { weakly in } W_{0}^{1, p}(\Omega), \\
u_{\varepsilon} \rightarrow u & \text { strongly in } L^{p q^{\prime}}(\Omega) .
\end{array}
$$

Recall that our assumptions on $q$ imply that $p q^{\prime}<p^{*}$.

Hence,

$$
\int_{\Omega} g(x)|u|^{p} \mathrm{~d} x=\lim _{\varepsilon \rightarrow 0^{+}} \int_{\Omega} g_{\varepsilon}(x)\left|u_{\varepsilon}\right|^{p} \mathrm{~d} x=1,
$$


and so

$$
\begin{aligned}
\lambda(g, V) & =\lim _{\varepsilon \rightarrow 0^{+}} \lambda_{\varepsilon} \\
& =\lim _{\varepsilon \rightarrow 0^{+}} \int_{\Omega}\left(\left|\nabla u_{\varepsilon}\right|^{2}+\varepsilon^{2}\right)^{(p-2) / 2}\left|\nabla u_{\varepsilon}\right|^{2}+V_{\varepsilon}(x)\left|u_{\varepsilon}\right|^{p} \mathrm{~d} x \\
& \geq \int_{\Omega}|\nabla u|^{p}+V(x)|u|^{p} \mathrm{~d} x \\
& \geq \lambda(g, V) .
\end{aligned}
$$

These imply that $u=u_{0}$, the unique normalized positive eigenfunction associated to $\lambda(g, V)$, and that $\left\|u_{\varepsilon}\right\|_{W_{0}^{1, p}(\Omega)} \rightarrow\|u\|_{W_{0}^{1, p}(\Omega)}$ as $\varepsilon \rightarrow 0^{+}$. So

$$
u_{\varepsilon} \rightarrow u_{0} \quad \text { strongly in } W_{0}^{1, p}(\Omega)
$$

This finishes the proof.

Remark 4.11. Observe that the eigenfunctions $u_{\varepsilon}$ are weak solutions to

$$
\begin{cases}-\operatorname{div}\left(\left(\left|\nabla u_{\varepsilon}\right|^{2}+\varepsilon^{2}\right)^{(p-2) / 2} \nabla u_{\varepsilon}\right)+V_{\varepsilon}(x)\left|u_{\varepsilon}\right|^{p-2} u_{\varepsilon}=\lambda_{\varepsilon} g_{\varepsilon}(x)\left|u_{\varepsilon}\right|^{p-2} u_{\varepsilon} & \text { in } \Omega \\ u=0 & \text { on } \partial \Omega\end{cases}
$$

Therefore, by the classical regularity theory (see [12]), the functions $u_{\varepsilon}$ are $C^{2, \delta}$ for some $\delta>0$.

With these preparatives we can now prove the following theorem.

Theorem 4.12. With the assumptions and notation of Theorem 4.8, we have that

$$
\left.\frac{\mathrm{d} \lambda(t)}{\mathrm{d} t}\right|_{t=0}=\lambda^{\prime}(0)=\int_{\Omega}(V(x)-\lambda(0) g(x)) \operatorname{div}\left(\left|u_{0}\right|^{p} W\right) \mathrm{d} x,
$$

for every field $W$ such that $\operatorname{div} W=0$.

Proof. During the proof of the theorem, we will require the eigenfunction $u_{0}$ to be $C^{2}$. As is well known (see [16]), this is not true. In order to overcome this difficulty, we regularize the problem and work with the regularized eigenfunctions $u_{\varepsilon}$ defined in Lemma 4.10 .

Since the eigenfunctions in the resulting formula only appear up to the first derivatives of $u_{\varepsilon}$ and $u_{\varepsilon} \rightarrow u_{0}$ strongly in $W_{0}^{1, p}(\Omega)$, the result will follow.

For the sake of simplicity, we choose to work formally with $u_{0}$. The changes in order to make this argument rigorous are straightforward.

Let $W \in C_{0}^{1}\left(\Omega ; \mathbb{R}^{N}\right)$. Then, we have that

$$
\int_{\Omega} \operatorname{div}\left(\left|\nabla u_{0}\right|^{p} W\right) \mathrm{d} x=0
$$


So,

$$
\begin{aligned}
p \int_{\Omega}\left|\nabla u_{0}\right|^{p-2}\left\langle\nabla u_{0},{ }^{T} W^{\prime} \nabla u_{0}^{T}\right\rangle \mathrm{d} x= & p \int_{\Omega}\left|\nabla u_{0}\right|^{p-2}\left\langle\nabla u_{0},{ }^{T} W^{\prime} \nabla u_{0}^{T}\right\rangle \mathrm{d} x \\
& +\int_{\Omega} \operatorname{div}\left(\left|\nabla u_{0}\right|^{p} W\right) \mathrm{d} x \\
= & p \int_{\Omega}\left|\nabla u_{0}\right|^{p-2}\left\langle\nabla u_{0},{ }^{T} W^{\prime} \nabla u_{0}^{T}\right\rangle \mathrm{d} x \\
& +p \int_{\Omega}\left|\nabla u_{0}\right|^{p-2}\left\langle\nabla u_{0}, D^{2} u_{0} W^{T}\right\rangle \mathrm{d} x \\
= & p \int_{\Omega}\left|\nabla u_{0}\right|^{p-2}\left\langle\nabla u_{0},{ }^{T} W^{\prime} \nabla u_{0}^{T}+D^{2} u_{0} W^{T}\right\rangle \mathrm{d} x \\
= & p \int_{\Omega}\left|\nabla u_{0}\right|^{p-2}\left\langle\nabla u_{0}, \nabla\left\langle\nabla u_{0}, W\right\rangle\right\rangle \mathrm{d} x .
\end{aligned}
$$

Now, we use the fact that $u_{0}$ is a weak solution to (1.1) to get

$$
\begin{aligned}
\lambda^{\prime}(0) & =p \int_{\Omega}(V(x)-\lambda(0) g(x))\left|u_{0}\right|^{p-2} u_{0}\left\langle\nabla u_{0}, W\right\rangle \mathrm{d} x \\
& =\int_{\Omega}(V(x)-\lambda(0) g(x)) \operatorname{div}\left(\left|u_{0}\right|^{p} W\right) \mathrm{d} x .
\end{aligned}
$$

The proof is now complete.

\section{REFERENCES}

[1] Mark S. Ashbaugh and Evans M. Harrell II. Maximal and minimal eigenvalues and their associated nonlinear equations. J. Math. Phys., 28(8):1770-1786, 1987. MR899179(88g:35149)

[2] G. R. Burton. Rearrangements of functions, maximization of convex functionals, and vortex rings. Math. Ann., 276(2):225-253, 1987. MR870963 (88d:49020)

[3] G. R. Burton. Variational problems on classes of rearrangements and multiple configurations for steady vortices. Ann. Inst. H. Poincaré Anal. Non Linéaire, 6(4):295-319, 1989. MR.998605 (90h:58017)

[4] Fabrizio Cuccu, Behrouz Emamizadeh, and Giovanni Porru. Nonlinear elastic membranes involving the $p$-Laplacian operator. Electron. J. Differential Equations, No. 49, 10 pp. (electronic), 2006. MR:2226922 (2006m:35090)

[5] Fabrizio Cuccu, Behrouz Emamizadeh, and Giovanni Porru. Optimization of the first eigenvalue in problems involving the $p$-Laplacian. Proc. Amer. Math. Soc., 137(5):1677-1687, 2009. MR2470826 (2009m:35353)

[6] Mabel Cuesta. Eigenvalue problems for the $p$-Laplacian with indefinite weights. Electron. J. Differential Equations, No. 33, 9 pp. (electronic), 2001. MR.1836801 (2002b:35165)

[7] Mabel Cuesta and Humberto Ramos Quoirin. A weighted eigenvalue problem for the p-Laplacian plus a potential. Technical report, L.M.P.A., May 2008. MR 2525513

[8] Leandro M. Del Pezzo and Julián Fernández Bonder. Some optimization problems for p-Laplacian type equations. Appl. Math. Optim., 59:365-381, 2009. MR.2491703

[9] Julián Fernández Bonder and Leandro M. Del Pezzo. An optimization problem for the first eigenvalue of the $p$-Laplacian plus a potential. Commun. Pure Appl. Anal., 5(4):675-690, 2006. MR2246002 (2007g:35050)

[10] Julián Fernández Bonder, Pablo Groisman, and Julio D. Rossi. Optimization of the first Steklov eigenvalue in domains with holes: A shape derivative approach. Ann. Mat. Pura Appl. (4), 186(2):341-358, 2007. MR 2295124(2007m:35179)

[11] A. Henrot and M. Pierre. Variation et Optimisation de forms: une analyse géométrique. Mathematics and Applications, volume 48. Springer-Verlag, 2005. MR2512810|(2009m:49003)

[12] Olga A. Ladyzhenskaya and Nina N. Ural'tseva. Linear and quasilinear elliptic equations. Translated from the Russian by Scripta Technica, Inc. Translation editor: Leon Ehrenpreis. Academic Press, New York, 1968. MR0244627 (39:5941) 
[13] Édouard Oudet. Numerical minimization of eigenmodes of a membrane with respect to the domain. ESAIM Control Optim. Calc. Var., 10(3):315-330 (electronic), 2004. MR2084326 (2005f:65144)

[14] Olivier Pironneau. Optimal shape design for elliptic systems. Springer Series in Computational Physics. Springer-Verlag, New York, 1984. MR725856 (86e:49003)

[15] James Serrin. Local behavior of solutions of quasi-linear equations. Acta Math., 111:247-302, 1964. MR0170096 (30:337)

[16] Peter Tolksdorf. Regularity for a more general class of quasilinear elliptic equations. J. Differential Equations, 51(1):126-150, 1984. MR727034 (85g:35047)

Departamento de Matemática, FCEyn, Universidad de Buenos Aires, Pabellón i, Ciudad Universitaria (1428), Buenos Aires, Argentina

E-mail address: ldpezzo@dm.uba.ar

Departamento de Matemática, FCEyn, Universidad de Buenos Aires, Pabellón I, Ciudad Universitaria (1428), Buenos Aires, Argentina

E-mail address: jfbonder@dm.uba.ar

$U R L:$ http://mate.dm.uba.ar/ j jfbonder 